with transvenous leads; aspirin and warfarin did not decrease the risk of systemic thromboemboli, but the study was underpowered to assess this issue.

The authors conclude that transvenous pacemakers or ICD leads seem to increase the risk of systemic thromboemboli in patients with intracardiac shunts. Before implantation of transvenous leads, the shunt should be eliminated in these patients, or an epicardial approach should be considered.

Original article Khairy P et al. (2006) Transvenous pacing leads and systemic thromboemboli in patients with intracardiac shunts: a multicenter study. Circulation 113: 2391-2397

\section{Management of patients with LV dysfunction needing permanent ventricular pacing support}

Right ventricular (RV) pacing is the standard approach for treating patients with symptomatic bradycardia and atrioventricular malconduction, but could induce heart failure in patients with left ventricular (LV) dysfunction. Results of trials evaluating biventricular (BV) stimulation have been inconsistent, leading to confusion regarding which is the best strategy.

In the prospective, randomized HOBIPACE trial, patients with LV dysfunction and a standard indication for permanent ventricular pacing were assigned to 3 months of RV or BV pacing. The BV group had lower LV end-diastolic $(-9.0 \%)$ and end-systolic $(-16.9 \%)$ volumes, $\mathrm{N}$-terminal pro-brain natriuretic peptide levels $(-31.0 \%$, reflecting reduced $L V$ wall stress) and Minnesota Living with Heart Failure score $(-18.9 \%)$ than patients receiving RV pacing. Additionally, LV ejection fraction, peak oxygen consumption, oxygen uptake at the ventilatory threshold and peak circulatory power were all higher in the BV group than in the LV group (by $22.1 \%, 12.0 \%, 12.5 \%$ and $21.0 \%$, respectively). BV pacing improved some other parameters of LV function that were unaffected by $\mathrm{RV}$ pacing, and showed greater efficacy than both RV septal and RV apical pacing.

The authors conclude that BV pacing is superior to RV pacing in this patient group as regards LV function, quality of life, and maximal and submaximal exercise capacity. Further studies are required to help identify the patients who would be most likely to benefit from BV pacing and, therefore, warrant the surgical skills and time required for the procedure despite its inherent risks.

Original article Kindermann M et al. (2006) Biventricular versus conventional right ventricular stimulation for patients with standard pacing indication and left ventricular dysfunction: the Homburg Biventricular Pacing Evaluation (HOBIPACE). J Am Coll Cardiol 47: 1927-1937

\section{Blood pressure and cardiovascular outcomes in high-risk Hispanics}

Hispanics have an increased likelihood of possessing certain risk factors for coronary artery disease (CAD), including hypertension, but clinical trial data guiding treatment of this group are lacking. Using information from the prospective, randomized INVEST trial, which compared atenolol and sustained-releaseverapamil strategies in a large cohort of patients with CAD and hypertension, Cooper-DeHoff et al. compared blood pressure control and risk of clinical adverse outcomes in Hispanic and non-Hispanic patients.

Antihypertensive treatment reduced blood pressure by a greater degree in Hispanics than in non-Hispanics, which probably contributed to the former's significantly lower risk of experiencing the combined endpoint of a nonfatal myocardial infarction, nonfatal stroke, or death $(P=0.009)$. Hispanic patients had a notably increased risk of new-onset diabetes during follow-up in INVEST. In these patients, the sustained-release-verapamil strategy was more effective than the atenolol strategy at reducing the risk of new-onset diabetes; this effect was attributed to the fact that the majority of patients on verapamil also took the angiotensinconverting-enzyme inhibitor trandolapril. Both the atenolol and the verapamil strategies were successful at lowering blood pressure.

Hypertension in Hispanics would, according to these results, best be treated with multiple drugs, including agents to help prevent or delay the development of diabetes. The authors recommend that a sustained-release-verapamil strategy be considered as an alternative to an atenolol strategy in Hispanic patients with hypertension and CAD.

Original article Cooper-DeHoff RM et al. (2006) Blood pressure control and cardiovascular outcomes in high-risk Hispanic patients - findings from the International Verapamil SR/Trandolapril Study (INVEST). Am Heart J 151: 1072-1079 